\title{
Abacavir use and myocardial infarction - Where are we now?
}

\author{
Satyajit Das* \\ Professor of Sexual Health and HIV, Integrated Sexual Health Service and Clinical Assessment Service, Coventry \& Warwickshire Partnership Trust, UK
}

The ongoing exposure of antiretrovirals of different classes and different side effect profiles have emerged with a variety of problems in the management of HIV infection. Drug related toxicity appears to be more common than AIDS or AIDS-related conditions in the era of highly active antiretroviral therapy (HAART). A significant body of evidence suggests there is increased risk of cardiovascular disease (CVD) in HIV patients [1,2]. There have been conflicting reports of increased risk of myocardial infarction (MI) in patients who have received abacavir. This has been observed in several cohort studies including a recent study from NA-ACCORD participants and a retrospective analysis of one randomised control trial [3-6].

This observation has been contradicted by several other observational cohort studies and meta-analysis conducted by GSK that did not show any association of abacavir with MI and cardiovascular disease $[7,8]$. In a recent pooled analysis of data from clinical trials, the incidents of cardiovascular events amongst abacavir users and abacavir non-users were found to be the same or similar [9]. This pooled analysis included 66 clinical trials where over 13,000 adults were on an abacavir containing antiretroviral regimen and over 7,000 were not on an abacavir containing regimen. This pooled analysis included randomised controlled trials and non-randomised controlled trials and of various durations and the results were unaffected by the design and duration of the trials included [9].

The first study which created a lot of interest about abacavir use and the risk of CVD came from D:A:D cohort [3]. The findings from $\mathrm{D}: \mathrm{A}: \mathrm{D}$ cohort suggested that use of abacavir was associated with an increased risk of MI both in terms of cumulative and recent exposure to the drug. Soon after, The ANRS cohort, French database demonstrated an increased risk only while on abacavir for the first year of therapy, but not when continued for more than one year [4].

Both ANRS and D:A:D cohort suggest that the risk is modifiable, and once abacavir was withdrawn the risk attenuates. Subsequently, the same group from the ANRS in France updated the results of their studies and these suggest that people with traditional cardiovascular risk factors were significantly more likely to receive either abacavir or tenofovir. Interestingly, when they controlled for the traditional CVD risk factors, cocaine abuse and intravenous drug use, the effect of abacavir alone on increased CVD risk in their cohort disappeared.

The Veterans Health Administration Clinical Case Registry (VA), a large American Study demonstrated an association between the use of abacavir and an increased risk of MI which was very consistent with other cohort data [7]. But they found that the patients with CKD had a significantly increased risk of acute $\mathrm{MI}$ and when they controlled for the presence of CKD, the effect of abacavir was no longer significant. Similarly, when they controlled for traditional CVD risk factors, the effect of abacavir was also attenuated and when traditional factors and CKD were combined the risk of CVD essentially disappeared. The VA study, not surprisingly, showed that patients with CKD were significantly more likely to have received abacavir than tenofovir because of the known association between tenofovir and renal disease.

The argument continues that abacavir is known to have a neutral lipid profile; it does not interfere with insulin sensitivity or insulin resistance and is not associated with increase in blood pressure and increase in visceral fat or wasting of lean fat. It is not known to have any interference or association with the known traditional risk factors for cardiovascular disease. The controversy has increased by the lack of definite mechanisms of action to justify the harmful actions of abacavir causing vascular changes or atherosclerosis or factors responsible for heart attacks. However, there has been a lot of research to find out what could be the mechanisms responsible for the association of abacavir with cardiovascular events. Some of the basic research has currently hinted or suggested that there may be a change of homeostatic mechanism responsible for abacavir and heart attacks (Table 1) [10]. Abacavir is a purine analogue and it has structural similarities to endogenous purines that are capable of triggering pro-inflammatory and pro-thrombotic process. Abacavir has been linked with enhanced platelet activity leading to platelet aggregation, enhanced recruitment of leucocytes by endothelium with involvement of $\mathrm{P} 2 \mathrm{X} 7$ receptor, a key phenomenon involved in inflammation and thrombi formation (Figure 1) [10]. Most of the evidence which has been put forward as a mechanistic process are in vitro or in animal models or from subjects who have not actually had myocardial infarction and also the numbers are very small, and their methodology has been questioned in different formats.

Abacavir has been used in many patients who have severe renal impairment even in patients who have end stage renal failure. But the cardiovascular events which have been found in this group of people

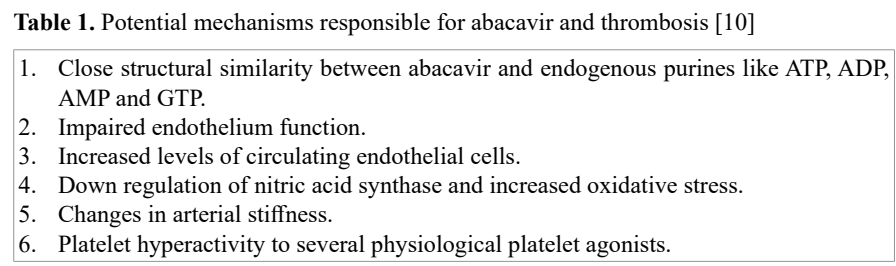

${ }^{\star}$ Correspondence to: Satyajit Das, Professor of Sexual Health and HIV, Integrated Sexual Health Service and Clinical Assessment Service, Coventry \& Warwickshire Partnership Trust, UK, E-mail: Satyajit.das@covwarkpt.nhs.uk

Received: June 12, 2019; Accepted: June 19, 2019; Published: June 21, 2019 


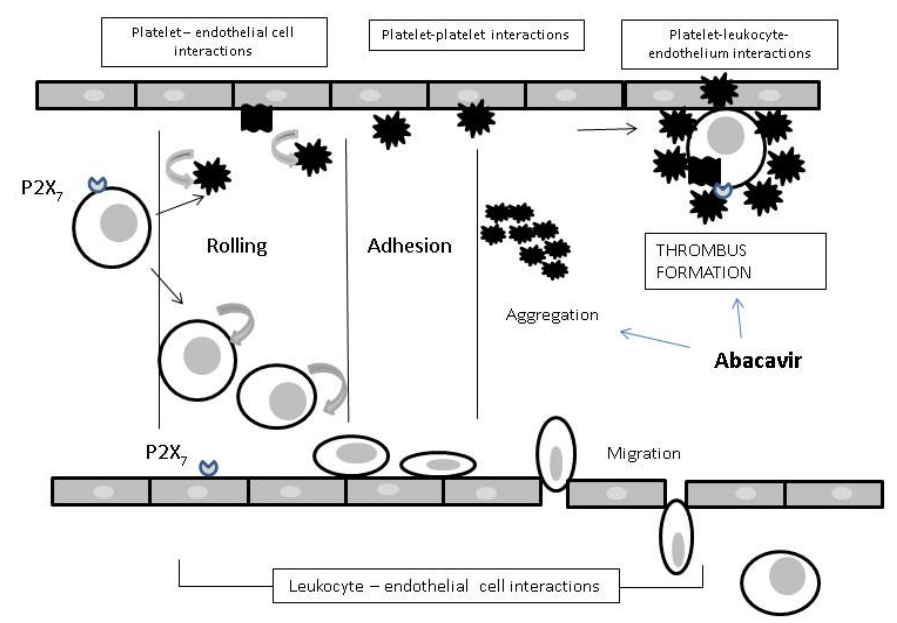

Figure 1. Abacavir and vascular inflammation and thrombosis [10]

are very small in number and abacavir has been used successfully. With a combination of abacavir with lamivudine and dolutegravir available as a single pill, it is likely that their use has been increased and it may continue to increase or remain as such in clinical practice.

Most of the guidelines, if not all, have recommended that abacavir should be used with caution and it should not be used in patients whose risk of cardiovascular disease is high $[11,12]$. The question still remains what drugs to be used in patients who have serious impairment of their renal function and if we have to use any nucleoside analogue, we have the option of using abacavir or tenofovir alfenamide fumarate (TAF). But TAF is not licenced to be used in serious renal impairment, particularly in patients who are on haemodialysis or peritoneal dialysis.

In spite of several recent studies, the impact of HIV and different antiretroviral agents on the risk of CVD in HIV-infected patients remains somewhat obscure. Differences in study design, endpoints, patient populations and limited follow-up in some studies prevent definitive comparisons. Several gaps in knowledge and controversies need to be addressed in future research. It is not clear what the clinical significance of an increase in relative CVD risk is among HIV-infected patients if the absolute risk of cardiovascular events remains low in this population. The assessment of traditional and non-traditional risk factors including drug-induced or HIV-related lipid abnormalities compared with those that arise naturally in the pathogenesis of atherosclerosis may not have similar impact. Unmeasured confounding factors such as intensity of smoking, intensity of cocaine use, concomitant infections and low socioeconomic status may yet have an effect on the association between HIV infection and risk of CVD in those patient populations. The inflammatory markers that are important in the general population including high sensitivity C-reactive protein (hs-CRP) may not have the same impact in an HIV-infected patient population. Nevertheless, risk of CVD in the HIV-infected population appears to be relatively higher than in the general population and the underlying cause appears to be multifactorial involving mechanisms which are specific to HIV infection and antiretroviral therapy in addition to the traditional CVD risk factors. The risks associated with modifiable risk factors including smoking, hypertension, insulin resistance and elevated lipids far outweigh the potential risks of antiretroviral use.

In the absence of any definite evidence it would be safer to consider abacavir to have a small but potentially increased risk for CVD, particularly in patients who already have a higher risk from traditional cardiovascular risk factors. Hence, assessment and modification of these underlying risk factors including hypertension, abnormal lipids, diabetes or raised insulin resistance and smoking should play an important role in the management of HIV infection. The choice of antiretroviral regimens should be individualised for patients to achieve maximum viral suppression as well as avoiding long-term toxicity.

\section{References}

1. Currier JS, Taylor A, Boyd F, Dezii CM, Kawabata H, et al. (2003) Coronary heart disease in HIV-infected individuals. J Acquir Immune Defic Syndr 33: 506-512. [Crossref]

2. Das S (2010) Risk of cardiovascular disease in HIV-infected patients. J Antimicrob Chemother 65: 386-9. [Crossref]

3. D:A:D Study Group, Sabin CA, Worm SW, Weber R, Reiss P et al. (2008) Use of nucleoside reverse transcriptase inhibitors and risk of myocardial infarction in HIVinfected patients enrolled in the D:A:D study: a multi-cohort collaboration. Lancet 372: 292. [Crossref]

4. Lang S, Mary-Krause M, Cotte L, Gilquin J, Partisani M, et al. (2010) Impact of Individual Antiretroviral Drugs on the Risk of Myocardial Infarction in Human Immunodeficiency Virus-Infected Patients: A Case-Control Study Nested Within the French Hospital Database on HIV ANRS Cohort CO4. Arch Intern Med 170: 12281238. [Crossref]

5. Martin A, Bloch M, Amin J, Baker D, Cooper DA, et al. (2009) Simplification of Antiretroviral Therapy with Tenofovir-Emtricitabine or Abacavir-Lamivudine: A Randomized, 96-Week Trial. Clin Infect Dis 49: 1591-1601. [Crossref]

6. Elion RA, Althoff KN, Zhang J, Moore RD, Gange SJ, et al (2018) Recent Abacavir Use Increases Risk of Type 1 and Type 2 Myocardial Infarctions Among Adults With HIV. J Acquir Immune Defic Syndr 78: 62-72. [Crossref]

7. Bedimo RJ, Westfall AO, Drechsler H, Vidiella G, Tebas P (2011) Abacavir use and risk of acute myocardial infarction and cerebrovascular events in the highly active antiretroviral therapy era. Clin Infect Dis 53: 84-91. [Crossref]

8. Brothers CH, Hernandez JE, Cutrell AG, Curtis L, Ait-Khaled M, et al. (2009) Risk of myocardial infarction and abacavir therapy: no increased risk across 52 GlaxoSmithKline-sponsored clinical trials in adult subjects. J Acquir Immune Defic Syndr 51: 20-28. [Crossref]

9. Nan C, Shaefer M, Urbaityte R, Oyee J, Hopking J, et al. (2018) Abacavir Use and Risk for Myocardial Infarction and Cardiovascular Events: Pooled Analysis of Data from Clinical Trials. Open Forum Infect Dis 5: ofy086. [Crossref]

10. Alvarez A, Orden S, Andújar I, Collado-Diaz V, Núñez-Delgado S, et al. (2017) Cardiovascular toxicity of abacavir: a clinical controversy in need of a pharmacological explanation. AIDS 31: 1781-1795. [Crossref]

11. Laura Waters, Ahmed N, Angus B, Boffito M, Bower M, et al. (2015) BHIVA guidelines for the treatment of HIV-1-positive adults with antiretroviral therapy 2015 (2016 interim update). https://www.bhiva.org/HIV-1-treatment-guidelines.

12. EACS guidelines - version 9.1 update (2018)1) http://www.eacsociety.org/files/2018 guidelines-9.1-english.pdf

Copyright: (C2019 Das S. This is an open-access article distributed under the terms of the Creative Commons Attribution License, which permits unrestricted use, distribution, and reproduction in any medium, provided the original author and source are credited. 